\title{
Computational Insights into the Activity of Transition Metals for Biomimetic $\mathrm{CO}_{2}$ Hydration
}

\author{
Manju Verma, K. B. Sravan Kumar, Parag A. Deshpande* \\ Quantum and Molecular Engineering Laboratory, \\ Department of Chemical Engineering, \\ Indian Institute of Technology Kharagpur, \\ Kharagpur 721302, India \\ Supporting information File
}

Table S1: Absolute DFT energies, important bond lengths and bond angles involved during CHR with different transition metals in vacuum

Table S2: Absolute DFT energies, important bond lengths and bond angles involved during CHR with different transition metals in water as solvent

* Email: parag@che.iitkgp.ernet.in

Tel: (+91) 3222-283916 
Table S1: Absolute DFT energies, important bond lengths and bond angles involved during CHR with different transition metals in vacuum

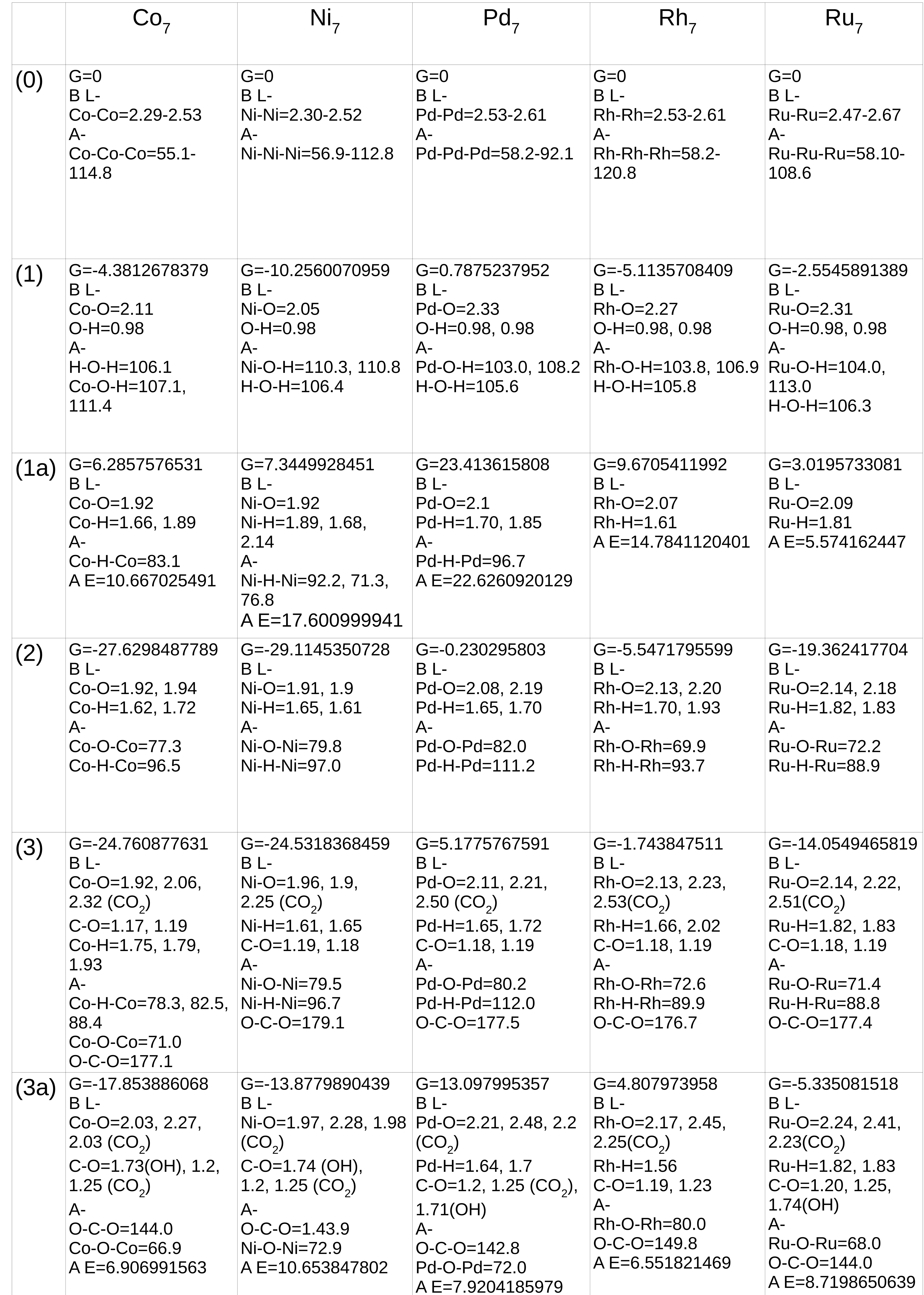

$\mathrm{G}=$ Relative Free Energy (kcal/mol), $\mathrm{B} L=$ Bond length $(\AA), A=$ Angle $\left(^{\circ}\right), A E=$ Activation Energy $(\mathrm{kcal} / \mathrm{mol})$ 
Table S1: Absolute DFT energies, important bond lengths and bond angles involved during CHR with different transition metals in vacuum

$$
\begin{array}{|l|l|l|l|l|}
\mathrm{Co}_{7} & \mathrm{Ni}_{7} & \mathrm{Pd}_{7} & \mathrm{Rh}_{7} & \mathrm{Ru}_{7} \\
\hline
\end{array}
$$

\begin{tabular}{|c|c|c|c|c|c|}
\hline (4) & $\begin{array}{l}\text { G=-21.78083739 } \\
\text { B L- } \\
\text { Co-O=1.91, 2.04 } \\
\text { C-O=1.22, 1.28, } 1.47 \\
\text { A- } \\
\text { O-C-O=111.5, 116.5, } \\
132.0\end{array}$ & $\begin{array}{l}\mathrm{G}=-22.0324684989 \\
\mathrm{~B} L- \\
\mathrm{Ni}-\mathrm{O}=1.87,1.97 \\
\mathrm{C}-\mathrm{O}=1.12,1.22,1.28 \\
\mathrm{~A}- \\
\mathrm{O}-\mathrm{C}-\mathrm{O}=112,116, \\
132\end{array}$ & $\begin{array}{l}\mathrm{G}=4.2959266142 \\
\mathrm{~B} L- \\
\mathrm{Pd}-\mathrm{O}=2.06,2.23 \\
\mathrm{C}-\mathrm{O}=1.23,1.29,1.43 \\
\mathrm{~A}- \\
\mathrm{O}-\mathrm{C}-\mathrm{O}=114.2,117.2 \\
128.6\end{array}$ & $\begin{array}{l}G=-8.8773698229 \\
\text { B L- } \\
\text { Rh-O=2.09, } 2.18 \\
C-O=1.22,1.28, \\
1.46 \\
\text { A- } \\
\text { O-C-O=113.2,115.8, } \\
131.0\end{array}$ & $\begin{array}{l}G=-18.367815939 \\
\text { B L- } \\
\text { Ru-O=2.21, } 2.05 \\
C-O=1.22,1.29,1.45 \\
\text { A- } \\
\text { O-C-O=111.8, } 117.3, \\
131.0\end{array}$ \\
\hline
\end{tabular}

(4a) $\begin{array}{ll}\text { G }=-8.099258663 & \mathrm{G}=0.353287567 \\ \text { B L- } & \text { B L- } \\ \text { Co-O=1.96, 1.97 } & \text { Ni-O=1.94,1.92 } \\ \text { C-O=1.26, 1.29, 1.39 } & \mathrm{C}-\mathrm{O}=1.22,1.26,1.29 \\ \mathrm{O}-\mathrm{H}=1.23,1.35 & \mathrm{O}-\mathrm{H}=1.22,1.35 \\ \text { A- } & \mathrm{A}=\end{array}$

$\begin{array}{ll}\mathrm{O}-\mathrm{C}-\mathrm{O}=104.1,123.9, & \mathrm{O}-\mathrm{C}-\mathrm{O}=124.5,129, \\ 132.0 & 131.3\end{array}$

$G=19.2419359761 \quad G=8.3282994481$

B L- $\quad$ B L-

$G=-0.6005261129$

$\mathrm{Pd}-\mathrm{O}=2.13,2.18 \quad \mathrm{Rh}-\mathrm{O}=2.13,2.15$

B L-

$\mathrm{Ru}-\mathrm{O}=2.11,2.14$

$\mathrm{C}-\mathrm{O}=1.26,1.29,1.38$

A- $\quad$ A- $\quad$ A-

$\mathrm{O}-\mathrm{C}-\mathrm{O}=104.1,126.4, \mathrm{O}-\mathrm{C}-\mathrm{O}=103.8,125.7, \mathrm{O}-\mathrm{C}-\mathrm{O}=104.6,124.2$, $129.6 \quad 130.5 \quad 131.2$

$\begin{array}{ll}132.0 & 131.3 \\ A E=13.681578727 & A E=22.385756066\end{array}$

A $E=14.9460093619$ A $E=17.2056692711$ A $E=17.7672898261$

(5) $G=-31.2424180919 \quad G=-32.7653824349$

B L- $\quad$ B L- $\quad$ B

$G=-7.4322165957 \quad G=-18.74996892 \quad G=-29.8549956931$

$B$ L-

$\begin{array}{llll}\mathrm{Co}-\mathrm{O}=1.95,1.97 & \mathrm{Ni}-\mathrm{O}=1.91,1.91 & \mathrm{Pd}-\mathrm{O}=2.12,2.16 & \mathrm{Rh}-\mathrm{O}=2.11,2.13 \\ \mathrm{C}-\mathrm{O}=1.27,1.28,1.36 & \mathrm{C}-\mathrm{O}=1.27,1.28,1.36 & \mathrm{C}-\mathrm{O}=1.27,1.28,1.36 & \mathrm{C}-\mathrm{O}=1.27,1.29\end{array}$

B L-

A- A- $\quad$ A- 1.36

$\mathrm{O}-\mathrm{C}-\mathrm{O}=115.3,117.1, \mathrm{O}-\mathrm{C}-\mathrm{O}=115.4,116.9, \mathrm{O}-\mathrm{C}-\mathrm{O}=114.3,116.5, \mathrm{~A}-$

127.6

127.7

129.2

$\mathrm{O}-\mathrm{C}-\mathrm{O}=115.1$,

$116.5,128.4$

Ru-O=2.08, 2.11

$\mathrm{C}-\mathrm{O}=1.28,1.28,1.35$

A-

$\mathrm{O}-\mathrm{C}-\mathrm{O}=115.5,117.7$, 126.8
(6) $G=-27.5401149919 \quad G=-35.0984608969 \quad G=-10.3576635539 \quad G=-18.6043868319 \quad G=-31.935815537$ B L- $\quad$ B L-
Co-O=1.97, 2.05, $\mathrm{Ni}-\mathrm{O}=1.92,1.98, \quad \mathrm{Pd}-\mathrm{O}=2.16,2.18$, $2.18\left(\mathrm{H}_{2} \mathrm{O}\right)$ $2.18\left(\mathrm{H}_{2} \mathrm{O}\right)$
A- A- 2.3 $\left(\mathrm{H}_{2} \mathrm{O}\right)$
A-
$B$ L- $\quad B$ L-
$\mathrm{Rh}-\mathrm{O}=2.13,2.22, \quad \mathrm{Ru}-\mathrm{O}=2.13,2.15$, $2.31\left(\mathrm{H}_{2} \mathrm{O}\right) \quad 2.34\left(\mathrm{H}_{2} \mathrm{O}\right)$
A-
A-
$\mathrm{O}-\mathrm{C}-\mathrm{O}=115.7,117.3, \mathrm{O}-\mathrm{C}-\mathrm{O}=115.6,117.1, \mathrm{O}-\mathrm{C}-\mathrm{O}=114.2,117.1, \mathrm{O}-\mathrm{C}-\mathrm{O}=114.8$, 127.1 127.3
128.8 $116.8,128.6$
$\mathrm{O}-\mathrm{C}-\mathrm{O}=115.5,117.8$, 126.7

(6a) G=-26.4087162649 G=-23.1375118479

$B$ L-

$B$ L-

$G=0.181350101$

$B$ L-

Co-O $=1.94,2.03$

$\mathrm{Ni}-\mathrm{O}=1.89,1.99$

$\mathrm{Pd}-\mathrm{O}=2.11,2.24$

$\mathrm{O}-\mathrm{H}=1.02,0.97$

$\mathrm{O}-\mathrm{H}=1.03,0.97$

$\mathrm{O}-\mathrm{H}=1.01,0.97$

$G=-15.3557727389$

B L-

$\mathrm{Rh}-\mathrm{O}=2.06,2.24$

$\mathrm{O}-\mathrm{H}=1.0,0.97$

$\mathrm{B} \mathrm{L-}$

$\mathrm{C}-\mathrm{O}=1.25,1.29,1.37 \mathrm{C}-\mathrm{O}=1.25,1.29,1.37 \mathrm{C}-\mathrm{O}=1.25,1.29,1.37$

AAAA-

$\mathrm{O}-\mathrm{C}-\mathrm{O}=114.6,116.6, \mathrm{O}-\mathrm{C}-\mathrm{O}=114.5,116.7, \mathrm{O}-\mathrm{C}-\mathrm{O}=114.7,117.7, \mathrm{O}-\mathrm{C}-\mathrm{O}=113.9$,

128.8

128.8

127.9

$117.5,128.6$

$A E=1.1313987271 \quad A E=11.960949049$

$A E=10.5390136549$

$A E=3.2486140929$

(7)

$\mathrm{G}=-29.748946672$

$G=-26.7896142279$

B L-

$G=-6.0083986749$

$B$ L-

Co-O=1.94, $1.95 \quad \mathrm{Ni}-\mathrm{O}=1.9,1.92$

$\mathrm{O}-\mathrm{H}=0.97,1.09 \quad \mathrm{O}-\mathrm{H}=1.08,0.97$

$\mathrm{C}-\mathrm{O}=1.26,1.29,1.36 \mathrm{C}-\mathrm{O}=1.26,1.29$,

A1.37

$\mathrm{O}-\mathrm{C}-\mathrm{O}=115.3,115.4, \mathrm{~A}-$

$\mathrm{O}-\mathrm{C}-\mathrm{O}=115,115.7$, 129.3

$\mathrm{Pd}-\mathrm{O}=2.12,2.12$

$\mathrm{O}-\mathrm{H}=1.06,0.98$

$G=-19.5851833988$

B L-

$\mathrm{Rh}-\mathrm{O}=2.05,2.17$

$\mathrm{O}-\mathrm{H}=1.04,0.98$

$\mathrm{Ru}-\mathrm{O}=2.06,2.21$

$\mathrm{O}-\mathrm{H}=1.02,0.97$

$\mathrm{C}-\mathrm{O}=1.26,1.29,1.37 \mathrm{C}-\mathrm{O}=1.25,1.30$, A1.37

$\mathrm{O}-\mathrm{C}-\mathrm{O}=114.7,115.9, \mathrm{~A}$ -

129.2

129.4

$\mathrm{O}-\mathrm{C}-\mathrm{O}=113.8$

$116.7,129.5$

$\mathrm{C}-\mathrm{O}=1.25,1.30,1.36$ A-

$\mathrm{O}-\mathrm{C}-\mathrm{O}=114.3,117.5$, 128.2

$A E=11.202290668$

$G=-24.975485709$

B L-

$\mathrm{Ru}-\mathrm{O}=2.07,2.12$

$\mathrm{O}-\mathrm{H}=1.08,1.84$

$\mathrm{C}-\mathrm{O}=1.26,1.29,1.36$ A-

$\mathrm{O}-\mathrm{C}-\mathrm{O}=114.9,116.2$, 128.9

$G=$ Relative Free Energy $(\mathrm{kcal} / \mathrm{mol}), B \mathrm{~L}=$ Bond length $(\AA), A=$ Angle $\left({ }^{\circ}\right), A E=$ Activation Energy $(\mathrm{kcal} / \mathrm{mol})$ 
Table S2: Absolute DFT energies, important bond lengths and bond angles involved during CHR with different transition metals in water as solvent

\begin{tabular}{|c|c|c|c|c|c|}
\hline & $\mathrm{CO}_{7}$ & $\mathrm{Ni}_{7}$ & $\mathrm{Pd}_{7}$ & $\mathrm{Rh}_{7}$ & $\mathrm{Ru}_{7}$ \\
\hline (0) & $\begin{array}{l}G=0 \\
B L- \\
\text { Co-Co=2.34-2.55 } \\
\text { A- } \\
\text { Co-Co-Co=56.2- } \\
113.0\end{array}$ & $\begin{array}{l}\mathrm{G}=0 \\
\mathrm{~B} L- \\
\mathrm{Ni}-\mathrm{Ni}=2.34-2.50 \\
\mathrm{~A}- \\
\mathrm{Ni}-\mathrm{Ni}-\mathrm{Ni}=56.2-110.0\end{array}$ & $\begin{array}{l}G=0 \\
B L- \\
P d-P d=2.60-2.82 \\
A- \\
P d-P d-P d=55.8- \\
120.2\end{array}$ & $\begin{array}{l}\text { G=0 } \\
\text { B L- } \\
\text { Rh-Rh=2.52-2.63 } \\
\text { A- } \\
\text { Rh-Rh-Rh=58.4- } \\
119.7\end{array}$ & $\begin{array}{l}G=0 \\
B \text { L- } \\
\text { Ru-Ru=2.43-2.74 } \\
\text { A- } \\
\text { Ru-Ru-Ru=57.3- } \\
111.9\end{array}$ \\
\hline (1) & $\begin{array}{l}\mathrm{G}=19.4640741618 \\
\text { B L- } \\
\text { Co-O=2.15 } \\
\mathrm{O}-\mathrm{H}=0.98,0.98 \\
\text { A- } \\
\text { Co-O-H=99.3, } 107.3 \\
\mathrm{H}-\mathrm{O}-\mathrm{H}=104.5\end{array}$ & $\begin{array}{l}\mathrm{G}=-0.5804458248 \\
\mathrm{~B} \text { L- } \\
\mathrm{Ni}-\mathrm{O}=2.08 \\
\mathrm{O}-\mathrm{H}=0.98,0.98 \\
\mathrm{~A}- \\
\mathrm{Ni}-\mathrm{O}-\mathrm{H}=106.9,108.0 \\
\mathrm{H}-\mathrm{O}-\mathrm{H}=105.2\end{array}$ & $\begin{array}{l}\mathrm{G}=4.5324975069 \\
\mathrm{~B} L- \\
\mathrm{Pd}-\mathrm{O}=2.37 \\
\mathrm{O}-\mathrm{H}=0.98,0.98 \\
\mathrm{~A}- \\
\mathrm{Pd}-\mathrm{O}-\mathrm{H}=96.2,105.7 \\
\mathrm{H}-\mathrm{O}-\mathrm{H}=104.8\end{array}$ & $\begin{array}{l}\text { G=0.4141559399 } \\
\text { B L- } \\
\text { Rh-O=2.26 } \\
O-H=0.98,0.98 \\
\text { A- } \\
\text { Rh-O-H=108.4, } \\
111.0 \\
H-O-H=105.2\end{array}$ & $\begin{array}{l}G=2.316763228 \\
\text { B L- } \\
\text { Ru-O=2.34 } \\
O-H=0.98,0.98 \\
\text { A- } \\
\text { Ru-O-H=99.7, } 110.4 \\
H-O-H=105.3\end{array}$ \\
\hline (1a) & $\begin{array}{l}G=21.8059377499 \\
B \text { L- } \\
\text { Co-O=2.05 } \\
\text { Co-H=1.75, } 1.89 \\
O-H=0.98,1.75 \\
\text { A- } \\
\text { Co-H-Co=81.8 } \\
\text { A E=2.3418635881 }\end{array}$ & $\begin{array}{l}\mathrm{G}=3.9012234532 \\
\mathrm{~B} \mathrm{~L}- \\
\mathrm{Ni}-\mathrm{O}=2.01 \\
\mathrm{Ni}-\mathrm{H}=1.75,1.89 \\
\mathrm{O}-\mathrm{H}=0.98,1.36 \\
\mathrm{~A}- \\
\mathrm{Ni}-\mathrm{H}-\mathrm{Ni}=92.6 \\
\mathrm{~A} E=4.481669278\end{array}$ & $\begin{array}{l}G=16.2411879379 \\
B \text { L- } \\
P d-O=2.27 \\
P d-H=1.74,1.85 \\
\text { A- } \\
P d-H-P d=95.9 \\
A E=11.708690431\end{array}$ & $\begin{array}{l}G=6.718738863 \\
B L- \\
R h-O=2.22 \\
R h-H=1.64 \\
A E=6.3045829231\end{array}$ & $\begin{array}{l}G=4.5889733171 \\
B \text { L- } \\
\text { Ru-O=2.30 } \\
\text { Ru-H=1.69 } \\
\text { A E=2.272210089 }\end{array}$ \\
\hline (2) & $\begin{array}{l}G=-32.976852968 \\
\text { B L- } \\
\text { Co-O=2.00, } 1.98 \\
\text { Co-H=1.65, } 1.76 \\
\text { A- } \\
\text { Co-O-Co=78.1 } \\
\text { Co-H-Co=84.1 }\end{array}$ & $\begin{array}{l}\mathrm{G}=-34.3410575337 \\
\mathrm{~B} \mathrm{~L}- \\
\mathrm{Ni}-\mathrm{O}=1.93,1.95 \\
\mathrm{Ni}-\mathrm{H}=1.63,1.66 \\
\mathrm{~A}- \\
\mathrm{Ni}-\mathrm{O}-\mathrm{Ni}=77.9 \\
\mathrm{Ni}-\mathrm{H}-\mathrm{Ni}=95.3\end{array}$ & $\begin{array}{l}\text { G=-7.006765494 } \\
\text { B L- } \\
\text { Pd-O=2.13, } 2.26 \\
P d-H=1.65,1.74 \\
\text { A- } \\
\text { Pd-O-Pd=79.4 } \\
P d-H-P d=110.8\end{array}$ & $\begin{array}{l}\text { G=-17.255869991 } \\
\text { B L- } \\
\text { Rh-O=2.21, } 2.24 \\
\text { Rh-H=1.63 } \\
\text { A- } \\
\text { Rh-O-Rh=72.2 }\end{array}$ & $\begin{array}{l}\text { G=-28.6972415879 } \\
\text { B L- } \\
\text { Ru-O=2.20, } 2.27 \\
\text { Ru-H=1.85, } 1.86 \\
\text { A- } \\
\text { Ru-O-Ru=70.0 } \\
\text { Ru-H-Ru=87.9 }\end{array}$ \\
\hline (3) & $\begin{array}{l}\mathrm{G}=-19.8368145081 \\
\text { B L- } \\
\text { Co-O=1.97, } 2.10, \\
2.37\left(\mathrm{CO}_{2}\right) \\
\mathrm{C}-\mathrm{O}=1.18,1.19 \\
\mathrm{Co}-\mathrm{H}=1.70,1.74 \\
\text { A- } \\
\text { Co-H-Co=80.3 } \\
\text { Co-O-Co=75.2 } \\
\text { O-C-O=179.2 }\end{array}$ & $\begin{array}{l}\mathrm{G}=-27.8406918028 \\
\mathrm{~B} \mathrm{~L}- \\
\mathrm{Ni}-\mathrm{O}=1.92,1.99 \\
2.28\left(\mathrm{CO}_{2}\right) \\
\mathrm{Ni}-\mathrm{H}=1.62,1.66 \\
\mathrm{C}-\mathrm{O}=1.18,1.19 \\
\mathrm{~A}- \\
\mathrm{Ni}-\mathrm{O}-\mathrm{Ni}=78.0 \\
\mathrm{Ni}-\mathrm{H}-\mathrm{Ni}=95.3 \\
\mathrm{O}-\mathrm{C}-\mathrm{O}=179.5\end{array}$ & $\begin{array}{l}\mathrm{G}=1.723139714 \\
\mathrm{~B} L- \\
\mathrm{Pd}-\mathrm{O}=2.15,2.25 \\
\mathrm{Pd}-\mathrm{H}=1.65,1.74 \\
\mathrm{C}-\mathrm{O}=1.18,1.18 \\
\mathrm{~A}- \\
\mathrm{Pd}-\mathrm{O}-\mathrm{Pd}=79.6 \\
\mathrm{Pd}-\mathrm{H}-\mathrm{Pd}=110.5 \\
\mathrm{O}-\mathrm{C}-\mathrm{O}=178.8\end{array}$ & $\begin{array}{l}\text { G=-8.7261401539 } \\
\text { B L- } \\
\text { Rh-O=2.19, } 2.26 \\
\text { Rh-H=1.62 } \\
\text { C-O=1.18, } 1.18 \\
\text { A- } \\
\text { Rh-O-Rh=73.0 } \\
\text { O-C-O=178.0 }\end{array}$ & $\begin{array}{l}\text { G=-21.9207718971 } \\
\text { B L- } \\
\text { Ru-O=2.22, } 2.26 \\
\text { Ru-H=1.85, } 1.86 \\
C-O=1.18,1.18 \\
\text { A- } \\
\text { Ru-O-Ru=69.9 } \\
\text { Ru-H-Ru=88.2 } \\
O-C-O=178.4\end{array}$ \\
\hline (3a) & $\begin{array}{l}\mathrm{G}=0.4612191149 \\
B \mathrm{~L}- \\
\mathrm{Co}-\mathrm{O}=2.02,2.10 \\
2.27\left(\mathrm{CO}_{2}\right) \\
\mathrm{C}-\mathrm{O}=1.19,1.21 \\
\text { A- } \\
\mathrm{O}-\mathrm{C}-\mathrm{O}=156.4 \\
\text { Co-O-Co=69.6 } \\
\text { A E }=20.298033623\end{array}$ & $\begin{array}{l}\mathrm{G}=-21.7833474257 \\
\mathrm{~B} \mathrm{~L}- \\
\mathrm{Ni}-\mathrm{O}=1.96,2.14 \\
2.15\left(\mathrm{CO}_{2}\right) \\
\mathrm{C}-\mathrm{O}=1.19,1.21 \\
\mathrm{~A}- \\
\mathrm{O}-\mathrm{C}-\mathrm{O}=156.3 \\
\mathrm{Ni}-\mathrm{O}-\mathrm{Ni}=75.5 \\
\mathrm{~A} E=6.057344377\end{array}$ & $\begin{array}{l}G=2.469247915 \\
B \text { L- } \\
P d-O=2.17,2.27 \\
P d-H=1.65,1.73 \\
C-O=1.20,1.20 \\
A- \\
O-C-O=158.0 \\
P d-O-P d=78.5 \\
A E=0.746108201\end{array}$ & $\begin{array}{l}\mathrm{G}=-6.408121908 \\
\mathrm{~B} L- \\
\mathrm{Rh}-\mathrm{O}=2.19,2.36 \\
2.52\left(\mathrm{CO}_{2}\right) \\
\mathrm{Rh}-\mathrm{H}=1.59 \\
\mathrm{C}-\mathrm{O}=1.19,1.20 \\
\mathrm{~A}- \\
\mathrm{Rh}-\mathrm{O}-\mathrm{Rh}=82.2 \\
\mathrm{O}-\mathrm{C}-\mathrm{O}=158.7 \\
\text { A E }=2.318018246\end{array}$ & $\begin{array}{l}G=-20.636888483 \\
B L- \\
\text { Ru-O=2.22, } 2.30 \\
R u-H=1.85,1.86 \\
C-O=1.20,1.20 \\
\text { A- } \\
\text { Ru-O-Ru=69.4 } \\
O-C-O=159.2 \\
\text { A E =1.2838834141 }\end{array}$ \\
\hline
\end{tabular}

$\mathrm{G}=$ Relative Free Energy $(\mathrm{kcal} / \mathrm{mol}), B \mathrm{~L}=$ Bond length $(\AA), A=$ Angle $\left({ }^{\circ}\right), A \mathrm{E}=$ Activation Energy $(\mathrm{kcal} / \mathrm{mol})$ 
Table S2: Absolute DFT energies, important bond lengths and bond angles involved during CHR with different transition metals in water as solvent

\begin{tabular}{l|l|l|l|l}
$\mathrm{CO}_{7}$ & $\mathrm{Ni}_{7}$ & $\mathrm{Pd}_{7}$ & $\mathrm{Rh}_{7}$ & $\mathrm{Ru}_{7}$ \\
\hline
\end{tabular}
(4) $G=-31.467693823 \quad G=-34.5901786068 \quad G=-10.2886375639 \quad G=-24.296520971 \quad G=-35.530814598$
$\mathrm{B}$ L-
B L-
$B$ L-
$\mathrm{B} \mathrm{L}-$
B L-
\begin{tabular}{ll|l|l} 
Co-O=1.99, $2.11 \quad \mathrm{Ni}-\mathrm{O}=1.91,2.07$ & $\mathrm{Pd}-\mathrm{O}=2.15,2.32$ & $\mathrm{Rh}-\mathrm{O}=2.21,2.29$ & $\mathrm{Ru}-\mathrm{O}=2.16,2.30$
\end{tabular}
$\mathrm{C}-\mathrm{O}=1.24,1.28,1.43 \mathrm{C}-\mathrm{O}=1.24,1.28,1.43 \mathrm{C}-\mathrm{O}=1.25,1.28,1.42 \mathrm{C}-\mathrm{O}=1.25,1.32,1.37 \mathrm{C}-\mathrm{O}=1.24,1.28$,
A-
A-
A-
A-
1.42
$\mathrm{O}-\mathrm{C}-\mathrm{O}=113.0,117.0, \mathrm{O}-\mathrm{C}-\mathrm{O}=113.8,116.9, \mathrm{O}-\mathrm{C}-\mathrm{O}=114.7,117.0, \mathrm{O}-\mathrm{C}-\mathrm{O}=103.4,127.3, \mathrm{~A}-$
130.0
129.3
128.2
129.3
$\mathrm{O}-\mathrm{C}-\mathrm{O}=113.5$,
$117.3,129.3$

(4a)

$\begin{array}{lll}\text { G=2.975647678 } & \text { G=-12.9072326209 } & \text { G=8.147576856 } \\ \text { B L- } & \text { B L- } & \text { B L- } \\ \text { Co-O=1.98, 2.02 } & \text { Ni-O=1.95, 2.02 } & \text { Pd-O=2.23, 2.30 }\end{array}$

$\mathrm{G}=-4.236313259$

B L-

$\mathrm{G}=-14.67743551$

$\mathrm{Rh}-\mathrm{O}=2.21,2.30 \quad \mathrm{Ru}-\mathrm{O}=2.21,2.23$

$\mathrm{C}-\mathrm{O}=1.25,1.34,1.35 \mathrm{C}-\mathrm{O}=1.26,1.31,1.36 \mathrm{C}-\mathrm{O}=1.26,1.32,1.35 \mathrm{C}-\mathrm{O}=1.25,1.32,1.37 \mathrm{C}-\mathrm{O}=1.26,1.31$,

$\mathrm{O}-\mathrm{H}=1.08,1.56 \quad \mathrm{O}-\mathrm{H}=1.25,1.32 \quad \mathrm{~A}-$

A- 1.36

A- $\quad \mathrm{A}=$

$\mathrm{O}-\mathrm{C}-\mathrm{O}=104.1,127.7, \mathrm{O}-\mathrm{C}-\mathrm{O}=103.4,127.3, \mathrm{~A}-$

$\mathrm{O}-\mathrm{C}-\mathrm{O}=104.4,117.3$, O-C-O= 104.3,

138.3

$126.5,129.1$

129.3

$\mathrm{O}-\mathrm{C}-\mathrm{O}=104.3$,

$A E=34.4433415011 \quad A E=21.6829459859$

A E=18.4362144199 AE=20.060207712 $126.4,129.4$

$A E=20.853379088$

(5)

$\mathrm{G}=-37.0249135271$

$G=-40.4046770008$

$B$ L-

$B$ L-

$\mathrm{G}=-16.3786124091$

$\mathrm{B}$ L-

$\mathrm{G}=-29.2230941301$

B L-

$G=-41.0723465771$

$\mathrm{Co}-\mathrm{O}=2.02,2.02$

$\mathrm{Ni}-\mathrm{O}=1.97,2.00 \quad \mathrm{Pd}-\mathrm{O}=2.20,2.25$

$\mathrm{Rh}-\mathrm{O}=2.17,2.25$

$\mathrm{B}$ L-

$\mathrm{Ru}-\mathrm{O}=2.17,2.19$

$\mathrm{C}-\mathrm{O}=1.27,1.28,1.37 \mathrm{C}-\mathrm{O}=1.27,1.28,1.37 \mathrm{C}-\mathrm{O}=1.27,1.27,1.38$

AA-

A-

A-

$\mathrm{C}-\mathrm{O}=1.27,1.28$,

$\mathrm{O}-\mathrm{C}-\mathrm{O}=114.8,117.4, \mathrm{O}-\mathrm{C}-\mathrm{O}=114.3,117.0$,

127.7

128.7

129.8

$\mathrm{O}-\mathrm{C}-\mathrm{O}=114.5,116.0, \mathrm{~A}$ -

1.37

129.4

$\mathrm{O}-\mathrm{C}-\mathrm{O}=114.6$,

$117.3,128.1$

(6)

$\mathrm{G}=-33.2579770001$
B L-
Co-O=2.05, 2.10,
$2.21\left(\mathrm{H}_{2} \mathrm{O}\right)$

$G=-38.0571658319$

$B$ L-

$G=-13.6577333851$

$B$ L-

$\mathrm{Ni}-\mathrm{O}=1.95,2.02$,

2.18 $\left(\mathrm{H}_{2} \mathrm{O}\right)$

A-

A- A- A-

$\mathrm{Pd}-\mathrm{O}=2.23,2.26$,

2.32 $\left(\mathrm{H}_{2} \mathrm{O}\right)$

A-

$G=-24.659221173$

B L-

$G=-36.883096493$

$\mathrm{Rh}-\mathrm{O}=2.16,2.40$,

$2.41\left(\mathrm{H}_{2} \mathrm{O}\right)$

A-

$B$ L-

$\mathrm{Ru}-\mathrm{O}=2.20,2.21$,

2.42 $\left(\mathrm{H}_{2} \mathrm{O}\right)$

A-

127.9

128.0

129.4

129.1

$117.5,127.8$

(6a) $G=-2.6556180881$

$\begin{array}{lll}\text { B L- } & \text { B L- } & \text { B L- } \\ \text { Co- }=1.99, & \mathrm{Ni}-\mathrm{O}=1.96, & \text { Pd-O=2.24, } \\ 2.11\left(\mathrm{H}_{2} \mathrm{O}\right) & 2.07\left(\mathrm{H}_{2} \mathrm{O}\right) & 2.38\left(\mathrm{H}_{2} \mathrm{O}\right) \\ \mathrm{O}-\mathrm{H}=1.0,0.97 & \mathrm{O}-\mathrm{H}=1.0,0.98 & \mathrm{O}-\mathrm{H}=1.0,0.98\end{array}$

$\mathrm{C}-\mathrm{O}=1.26,1.28,1.38 \mathrm{C}-\mathrm{O}=1.26,1.28,1.38 \mathrm{C}-\mathrm{O}=1.26,1.28,1.39$

A- $\quad$ AA- $\quad$ A-

A- $\quad$ A-

$\mathrm{G}=-23.9149954991$

B L-

$G=-31.502206818$

$\mathrm{Rh}-\mathrm{O}=2.15,2.57$,

2.42 $\left(\mathrm{H}_{2} \mathrm{O}\right)$

$B$ L-

$\mathrm{O}-\mathrm{H}=0.99,0.98 \quad \mathrm{O}-\mathrm{H}=1.01,0.98$

$\mathrm{O}-\mathrm{C}-\mathrm{O}=115.5,116.1, \mathrm{O}-\mathrm{C}-\mathrm{O}=115.3,116.0, \mathrm{O}-\mathrm{C}-\mathrm{O}=115.3,115.4$,

128.4

128.7

129.2

A-

$\mathrm{C}-\mathrm{O}=1.26,1.28$

$A E=30.602358912$

$A E=7.3851534211 \quad A E=6.2976803241$

129.1 1.38

(7)

$G=-25.547146408$

B L-

$G=-36.1514209989$

B L-

$\mathrm{Co}-\mathrm{O}=2.03$,

$\mathrm{Ni}-\mathrm{O}=1.96$,

$1.96\left(\mathrm{H}_{2} \mathrm{O}\right)$

$G=-13.399199677$

$B$ L-

$2.06\left(\mathrm{H}_{2} \mathrm{O}\right)$

2.12 $\left(\mathrm{H}_{2} \mathrm{O}\right)$

$A E=0.744225674$

A-

$\mathrm{O}-\mathrm{H}=1.03,0.98$

$\mathrm{O}-\mathrm{H}=1.04,0.98$

$\mathrm{Pd}-\mathrm{O}=2.12$,

$\mathrm{G}=-28.7443047632$

$\mathrm{B}$ L-

$\mathrm{Rh}-\mathrm{O}=2.13$,

2.27 $\left(\mathrm{H}_{2} \mathrm{O}\right)$

$\mathrm{O}-\mathrm{H}=1.02,0.98$

$\mathrm{O}-\mathrm{C}-\mathrm{O}=115.5$,

115.6,129.0

$A E=5.380889675$

$\mathrm{C}-\mathrm{O}=1.26,1.28,1.39$ A-

$\mathrm{O}-\mathrm{H}=1.06,0.98$

$\mathrm{O}-\mathrm{H}=1.02,0.98$
$\mathrm{C}-\mathrm{O}=114.9,115.3$

AA129.9

$\mathrm{O}-\mathrm{C}-\mathrm{O}=114.4,115.5, \mathrm{O}-\mathrm{C}-\mathrm{O}=114.6,115.3, \mathrm{O}-\mathrm{C}-\mathrm{O}=114.7,115.9, \mathrm{~A}-$

130.1

130.1

129.4

$\mathrm{O}-\mathrm{C}-\mathrm{O}=115.4,115.6, \mathrm{O}-\mathrm{C}-\mathrm{O}=114.9$,

129.0

$115.6,129.5$

$\mathrm{G}=$ Relative Free Energy $(\mathrm{kcal} / \mathrm{mol}), \mathrm{B} L=$ Bond length $(\AA), A=$ Angle $\left({ }^{\circ}\right), A \mathrm{E}=$ Activation Energy $(\mathrm{kcal} / \mathrm{mol})$ 